\title{
Conceptos básicos sobre obesidad sarcopénica en el adulto mayor.
}

\author{
${ }^{1}$ Médico General, Docente en el Departamento de Anatomía de la Universidad de Costa Rica. drrafaelzuniga@gmail.com \\ ${ }^{2}$ Médico Especialista en Geriatría y Gerontología. Máster en Nutrición. Grupo Clínico de Soporte Nutricional, Hospital \\ Nacional de Geriatría y Gerontología.
}

\section{RESUMEN}

La obesidad sarcopénica es una entidad clínica y fisiopatológica que implica discapacidad y riesgo de mortalidad. Su definición ha sidosujeto de consensopor "The European Working Group on Sarcopenia in Older People" (EWGSOP) y el "International Working Group on Sarcopenia" (IWGS). Gracias a los estudios realizados por Baumgartner y Janssen la estandarización del diagnóstico de sarcopenia ha sido determinada empleando el índice de masa apendicular en relación a talla( ASM/ht ${ }^{2}$ ) y peso (ASM/Wt); basados en los análisis por densitometría dual por rayos X (DXA), e impedancia bioeléctrica (BIA). La obesidad se valora a través del índice de masa corporal y del porcentaje de grasa corporal. Metaanálisis recientes han estimado la incidencia de la obesidad sarcopénica entre el 2 y $20 \%$. La obesidad sarcopénica se ha caracterizado por la resistencia a insulina e inflamación. El adipocito ha sido implicado en el mantenimiento de este proceso. Estudios han sugerido un abordaje e intervención basados en la composición corporal y el riesgo metabólico.

\section{PALABRAS CLAVE}

Adulto mayor, obesidad sarcopénica, critérios diagnósticos

\footnotetext{
ABSTRACT

Sarcopenic Obesity is a clinical and pathophysiological entity that implies disability and risk of mortality. Its definition has been subject of consensus by The European Working Group on Sarcopenia in Older People (EWGSOP) and the International Working Group on Sarcopenia (IWGS). Thanks to the studies conducted by Baumgartner and Janssen, the standardization of sarcopenia's diagnosis has been determinated by the measurement of the appendicular muscle mass index using the height (ASM/ht 2) and body weight (ASM/Wt) analyzed on the Dual-energy X-ray Absorptiometry (DXA) and the Bioelectrical Impedance Analysis (BIA). Obesity is assessed by the body mass index and the body fat percentage. Recent meta-analysis has estimated an incidence of Sarcopenic Obesity from 2 to $20 \%$. Sarcopenic Obesity has been characterized by insulin,
}

resistance and inflammation. The adipocyte has been implicated in maintaining this process. There have been studies that suggest an approach and intervention based on the body composition and the metabolic risk.

\section{KEYWORDS}

Elderly, sarcopenic obesity, diagnostic criteria

\author{
ABREVIATURAS \\ BIA Análisis de bioimpedancia. \\ DXA Densitometría por absoción de rayos X. \\ TAC Tomografía Axial Computarizada. \\ RMN Resonancia Magnética Nuclear. \\ IMC Índice de masa corporal. \\ ASM/Wt Masa muscular apendicular en relación a peso. \\ $\mathrm{ASM} / \mathrm{Ht}^{2}$ Masa muscular apendicular en relación a \\ estatura. \\ EWGSOP "The European Working Group on Sarcopenia \\ in Older People". \\ IWGS "International Working Group on Sarcopenia". \\ CA: Circunferencia abdominal
}

\section{Introducción}

El presente trabajo busca hacer una revisión de los conceptos más recientes sobre obesidad sarcopénica recopilados por consensos y recomendaciones de expertos, con el fin de comprenderle como entidad clínica. Para esto se revisará la definición, epidemiología, fisiopatología y diagnóstico; de manera que permita establecer, en forma básica, su abordaje y la relevancia a través de la evidencia- de intervenir esta entidad en el adulto mayor.

\section{Metodología}

Se realizó una búsqueda de artículos en la base de datos Pub-Med, utilizando como criterio de búsqueda "avanzada" los términos "sarcopenia obesity ", "sarcopenic obesity" y "elderly". Se emplearon los siguientes filtros: idioma en español o inglés, ensayos clínicos y de revisión; en pacientes de 65 años o mayores, uno o ambos sexos. Se aplicaron como criterios de exclusión: aquellos que no cuenten con acceso un 
resumen del contenido. Además se consultó en forma manual las bases de Datos de MDConsult, Scielo, Springer link, Science Direct y Oxford Journals.

\section{Definición}

El término obesidad sarcopénica surge en 1996, por Heber y colaboradores, quienes la describen como "la masa (magra) reducida fuera de proporción en relación al tejido adiposo" -este último en exceso- .Otra fuente atribuye la conceptualización a Roubenoff en 2004 quien además define la masa magra como tejido óseo y músculo-esquelético y agrega que ambos se encuentran disminuidos en esta patología ${ }^{(1,2)}$. Este concepto surge a partir de la evolución de la comprensión y definición de la sarcopenia, sobre la cual se hará una reseña para comprender su origen y diagnóstico.

Primer componente, la conceptualización de la sarcopenia

Se remonta a 1989 cuando Irwin Rosenberg, propuso este término como la disminución de masa muscular en relación a la edad; la etimología proviene del griego "sarx" o carne y "penia" o pérdida ${ }^{(3,4)}$.

Consenso en la definición de sarcopenia por EWGSOP y la IWGS

En 2010 y en 2011 se conforman la "The European Working Group on Sarcopenia in Older People" (EWGSOP) y la "International Working Group on Sarcopenia" (IWGS); estos definen sarcopenia como "un sindrome donde hay una pérdida progresiva y generalizada de masa muscular esquelética, con una pérdida concomitante de fuerza, los cuales establecen un fenotipo de riesgo para discapacidad o limitación física, pobre calidad de vida y muerte". Es de importancia reconocer que la evolución del término implica que se da la connotación a la pérdida funcional. En este contexto, el consenso sugiere que la sarcopenia se defina como síndrome geriátrico dadas sus implicaciones clínicasepidemiológicas. ${ }^{(1,4,6)}$

La EWGSOP, propone como diagnóstico de sarcopenia con 2 de 3 criterios, siendo estos: 1) disminución de la masa muscular 2) disminución de fuerza 3) disminución del desempeño físico. Se requierela presencia del criterio 1 más el criterio 2 ó 3 o ambos.

La masa muscular se puede estimar por DXA (en inglés denominada "dual energy X-ray absorptiometry"), análisis de BIA (en inglés denominada "Bioimpedance analysis") o antropometría (por ejemplo la medición de la circunferencia del gastronemios o "pantorrilla") como métodos indirectos -siendo estos últimos menos empleados dado su poco uso en ensayos clínicos- ${ }^{(4,8)}$. La validación del uso de estos equipos y la determinación de los parámetros diagnósticos, proviene del estudio clínico de Baumgartner en la población de Nuevo México. En este se abordó la composición corporal a través de la DXA, en relación con distintos parámetros de morbilidad y funcionalidad -como actividades instrumentales de la vida diaria y marcha entre otros-. Para definir sarcopenia en la muestra seleccionada $(n=$ 301) del estudio denominado "The Aging Process Study"-estudio longitudinal en pacientes ancianos-, Baumgartner lo comparó con una muestra del estudio "Rossetta" (n=229) con individuos de 18 a 40 años. Se determinó el porcentaje de grasa corporal y de masa muscular apendicular (ASM) en ambos grupos. Se estandarizó la definición de sarcopenia usando el índice de ASM ajustados a la altura en metros cuadrados $\left(\mathrm{Ht}^{2}\right)$, es decir la relación $\mathrm{ASM} / \mathrm{Ht}^{2}$. Así quienes tuvieran un $\mathrm{ASM} / \mathrm{Ht}^{2}$ por debajo de las dos desviaciones estándar del grupo referencia, se catalogaron como sarcopénicos. La obesidad la correspondió con porcentajes de grasa corporal superiores a la media del control; $38 \%$ mujeres y $27 \%$ en hombres. Dentro de sus resultados se encontró que la definición empleada para sarcopenia correlacionó en forma efectiva en la predicción de limitación funcional y permitió objetivar un umbral diagnóstico empleando DXA ${ }^{(3)}$. En el caso del uso de BIA, el método fue validado a través del estudio de Janssen, en donde se empleó un índice modificado en su denominador, se empleó el peso (Wt: "weight") en lugar de la estatura dando lugar al índice ASM/Wt. ${ }^{(16)}$

La fuerza se puede cuantificar a partir de la prueba de prensión palmar por dinamómetro (en inglés denominada "handgrip test") con adecuada correlación clínica con estudios de dinamometría miembros inferiores, con flexo- extensión de rodilla. El desempeño físico se puede objetivar a través de tests como el "Short Physical Performance Battery", "Usual gait speed" "Get-up-andgo-test". Los puntos de corte ya han sido caracterizados en algunas poblaciones. ${ }^{(1,2,4-7)}$

\section{El segundo componente, la obesidad}

La obesidad, entendida como presencia de tejido adiposo en exceso, se ha definido a partir de los criterios antropométricos de índice de masa corporal (IMC) y circunferencia abdominal (CA). No obstante, en el caso del adulto mayor, los criterios diagnósticos no han sido tan claramente validados dado los factores de posible confusión a la hora de estimarlos. En la determinación del IMC se tiene que respecto al adulto de edad media, el adulto mayor es susceptible a un cambio de composición corporal: disminución de la altura, pérdida de peso magro, redistribución de tejido graso. Así se sugiere que, en lugar de emplearse el valor de 30 para el diagnóstico de obesidad, se emplee el valor de 27 para el diagnóstico del continuum sobrepeso-obesidad según los criterios de Lipschitz. En el caso de la circunferencia abdominal ésta presenta la variabilidad según el grupo étnico y por la redistribución de tejido graso; a nivel de criterios clásicos, -heredados de literatura 
norteamericana- se han empleado como puntos de corte para CA en obesidad un valor mayor o igual a $102 \mathrm{~cm}$ en hombres y mayor a $88 \mathrm{~cm}$ en mujeres. No obstante esto se debería estandarizar según cada población como por ejemplo en el caso de estudios como el KHANES ${ }^{(2)}$, se abordó la prevalencia de sarcopenia y obesidad sarcopénica en Korea y se usaron valores de CA específicos para su población. ${ }^{(2,8,9-11)}$

Un criterio propuesto es la medición del porcentaje de grasa corporal, en distintos estudios se reportan valores en mujeres entre 35 a $40 \%$ y en hombres entre 27 a $30 \%$. Dicha estimación ha sido determinada a través de DXA, BIA, TAC, RMN ${ }^{(3,8)}$. Además se proponen otros índices como el FMI (índice de grasa/altura) basado en los datos del estudio NHANES donde se determinó la composición corporal por DXA e IMC. Y a partir de este índice se desarrollaron valores estandarizados según sexo para clasificar el contenido de grasa corporal desde el déficit hasta la obesidad, graduada en tres niveles. ${ }^{(12)}$

Diagnóstico de sarcopenia en pacientes obesos

Cuando se aborda al paciente con aumento de tejido graso y disminución concomitante de masa muscular o presencia de debilidad, los criterios de definición diagnóstica del binomio obesidad y sarcopenia son heterogéneos respecto a metodología y por ende en la prevalencia de sarcopenia en pacientes obesos.En un metaanálisis de estudios que abordaron la obesidad sarcopénica, para una muestra total de alrededor de 23 000 pacientes. La DXA fue el método de mayor uso en la determinación de sarcopenia, seguido de TAC, BIA, y dinamómetro. ${ }^{(2,4-6)}$

\section{Obesidad sarcopénica como predictor de morbilidad}

El estudio de la obesidad sarcopénica toma importancia gracias a ensayos clínicos que han estudiado la composición corporal y su impacto en la funcionalidad. Se trató de una cohorte de 451 pacientes adultos mayores, en un periodo de 8 años, donde se dio seguimiento a 9 actividades instrumentales de la vida diaria. Como criterio de inclusión los pacientes tenían que tener un basal funcional alto, definido como el adecuado desempeño en al menos 8 tareas. El objetivo del estudio era medir la incidencia del deterioro funcional en la población estudiada según la antropometría. Se definió el deterioro como la pérdida de funcionalidad de 2 o más actividades instrumentales. El estudio concluye que la obesidad aumento en hombres 1,34 veces y en mujeres 2,15 veces el deterioro funcional, respecto a los que tenían una composición normal. En el caso de sarcopenia fue de 3,78 veces en hombres y 2.96 en mujeres, respecto al control. En obesidad sarcopénica se observó el mayor compromiso con un riesgo de 8,72 veces en hombres y 11.98 en mujeres. Además se determinó que el deterioro en el desempeño en actividades instrumentales correlacionaba con un aumento de 5 veces el riesgo de mortalidad. ${ }^{(2,13)}$
Del ensayo clínico EPIDOS, en una muestra de 1308 mujeres se determinó la limitación funcional en relación a la composición corporal en distintas tareas. Se analizaron pacientes con criterios para sarcopenia, obesidad, obesidad sarcopénica y pacientes dentro de parámetros normales partiendo de la antropometría. Se observó que la obesidad sarcopénica implicaba una mayor predisposición a la discapacidad en funciones físicas. Algunos hallazgos fueron no esperados; en ciertas tareas se observó que el paciente clasificado como sarcopénico tenía mejor desempeño que aquel clasificado como normal. Los autores propusieron la hipótesis de que el paciente sarcopénico tenía un fenotipo "relativamente" proporcionado, lo que le permitía desempeñar estas funciones mejor que los controles. El factor compensatorio en el paciente con sarcopenia era la asociación presente en este estudio entre un bajo nivel de masa muscular y grasa corporal. ${ }^{(14)}$

Aunque enla literatura se tiene que tanto la obesidad como la sarcopenia son factores predictores independientes de la limitación funcional en el adulto mayor; las implicaciones en el paciente obeso y sarcopénico tienden a ser mayores, esto porque se ha documentado una mayor limitación -efecto sinérgicocuando ambos factores coexisten. ${ }^{(2,7,15)}$

\section{Epidemiología de la obesidad sarcopénica}

La prevalencia de esta entidad está sujeta a una gran variabilidad según el diseño del estudio, su método de medición y los valores de referencia entre otros. Se reporta incidencia de sarcopenia entre el $4 \%$ y $12 \%$ y la obesidad sarcopénica podría rondar entre el $4 \%$ y $9 \%$. (7) Sin embargo otras revisiones sugieren datos muy divergentes, por ejemplo en una revisión sistemática se recopila información de prevalencia de obesidad sarcopénica que va de un 2.75 hasta un $20 \%{ }^{\left({ }^{(6)}\right.}$ Otros estudios reportan prevalencias de hasta un $50 \%{ }^{(16)}$

\section{Fisiología y Fisiopatología}

Dentro del estudio de la historia natural, la evolución hacia la obesidad sarcopénica no ha sido bien caracterizada. Se tiene la hipótesis de la ganancia peso en forma de tejido adiposo en individuos con peso normal o la pérdida excesiva de masa magra en pacientes obesos. Esto lleva a un cambio de composición corporal; donde se postula que el desbalance entre tejido graso y muscular explican el riesgo metabólico en función de una carga y una capacidad metabólica respectivamente. Sin embargo estudios propiamente sobre la su etiología, no han sido abordados en la literatura. ${ }^{(17)}$

Se han caracterizado los cambios de la composición corporal en relación con el envejecimiento. ${ }^{(15,18)}$ Aunque el peso tiende a mantenerse estable o disminuir en la tercera edad, se produce un cambio en la proporción de sus componentes. Mientras que el tejido graso se 
redistribuye $y$ se tiende a localizar a nivel intraabdominal e infiltrando tejidos, el tejido muscular y óseo disminuyen. Se documenta la pérdida de tejido muscular a edades tan tempranas como los 30 años. Además cabe resaltar que el proceso no es simétrico; por ejemplo, el compromiso en miembros inferiores suele ser mayor que los miembros superiores-

El cambio de composición corporal se atribuye a un proceso multifactorial que incluye factores genéticos, hormonales, inflamatorios, dietéticos, de estilo de vida. Se ha estudiado a su vez su interacción y preponderancia. En el caso del componente genético y estilos de vida, se denota un aporte modesto del primero, mientras que el segundo tiene una mayor preponderancia aunque su interacción es compleja. En el componente hormonal, median un déficit o resistencia a testosterona, déficit de estrógenos, hormona de crecimiento, un aumento de leptina, la resistencia insulina, entre otros. En este proceso de modulación endocrina intervienen también los procesos inflamatorios como los mediados por el tejido adiposo quienes actuarían a través de interleucinas y el factor de necrosis tumoral que disminuyen el tejido magro. En el caso del factor nutricional, el déficit de vitamina $\mathrm{D}$ se ha correlacionado con el proceso de cambio de composición corporal. (15,19-23) Además la pérdida fuerza muscular es hasta 3 veces mayor que la pérdida de masa muscular; a nivel neurológicohay cambios desde la vía piramidal hasta la composición tendinosa y cambios postraduccionales en las cadenas de miosina ${ }^{(19,21)}$.

El tejido graso actúa como determinante funcional en las distintas etapas de la vida. Así la obesidad en la infancia se correlaciona con la aparición de dolor; en relación a procesos migrañosos, esto aunado a la sintomatología emocional negativa y la disminución funcional en actividades recreativas. En el adulto se correlaciona con la dificultad en el desempeño del rol laboral, labores domésticas y un aumento de 5 puntos en el IMC correlaciona con un aumento del riesgo de osteoartrosis en $35 \%$. La obesidad interfiere, mediante el efecto mecánico así como por el proceso inflamatorio mediado por adipocinas, a nivel de articular. En el adulto mayor se tiene la hipótesis de un ciclo vicioso entre sedentarismo, obesidad y sarcopenia. ${ }^{(24)}$

En relación al proceso de obesidad y el síndrome metabólico, las interacciones han sido estudiadas. Así el proceso de insulino resistencia, la infiltración grasa de tejido muscular, hepático, inclusive pancreático corresponden a un mismo fenotipo que, aunado a la hipertensión, la dislipidemia -ambos relacionados a la arteriosclerosis- constituyen los desencadenantes de las cascadas de disregulación del metabolismo muscular. A nivel de estudios clínicos el efecto del tejido adiposo, el cambio hormonal y la aterosclerosis en relación corporal han evidenciado que estos son factores predisponentes a la pérdida de masa magra y además han denotado la interacción del género y la etnia en la presentación, definiendo grupos de riesgo. ${ }^{(17,18,24,25)}$

El estudio de la composición corporal y la antropometría en la práctica médica se justifica desde el punto de vista tanto diagnóstico como terapéutico. Para el adulto mayor, tanto la obesidad como la sarcopenia y recientemente la obesidad sarcopénica han sido fuente de estudio para la predicción de limitación funcional e inclusive mortalidad. $(1,2,4-6,17)$

La principal comorbilidad de la obesidad es el síndrome metabólico que se caracteriza por compromiso cardiovascular -incluyendo la aterosclerosis-, resistencia a la insulina, dislipidemia, deterioro cognitivo, compromiso respiratorio -apnea obstructiva del sueño-, incontinencia urinaria, disfunción sexual, osteoartrosis e inclusive cáncer. Además el compromiso, músculo esquelético derivado de la obesidad es fuente de dolor crónico así como dificultades en el desempeño de las actividades cotidianas, el desarrollo de fragilidad y deterioro de la calidad de vida. ${ }^{(2,25)}$

Strandberg y colaboradores abordaron el tema de la composición corporal en forma longitudinal, por medio de la cohorte del estudio en comerciantes de Helsinsky. ${ }^{(26)}$ En este estudio se valora el impacto de la trayectoria del IMC respecto a la edad (a partir del IMC a los 25 años hasta la tercera edad) y se compara mortalidad y morbilidad- esta última definida como fragilidad-. El estudio concluye, respecto a la variable mortalidad, que existe un riesgo relativo de $30 \%$ en pacientes con sobrepeso en forma constante y de un $80 \%$ o más en el grupo donde se documenta pérdida de peso, en comparación con los pacientes que mantienen un peso normal. Los que ganan peso después de la mediana edad tienen un riesgo comparable con los que se mantienen con peso normal. Además en los que tuvieron pérdida de peso, se vio que la relación se atenúa cuando se toma en cuenta el desarrollo de fragilidad, siendo esta última por tanto mejor predictor. Respecto al componente de morbilidad, en este estudio, se observa que los pacientes que tuvieron sobrepeso en forma constante tienen una tendencia mayor a desarrollar fragilidad cuando se comparan con los grupos de peso normal constante y el de ganancia de peso después de la mediana edad. Los pacientes con mayor tendencia a desarrollar fragilidad eran aquellos que perdían peso. El presente estudio con aproximadamente 1000 pacientes señala que se debe tener en consideración la tendencia de cambio en el peso, lo cual correlaciona con la relevancia del estudio de la composición y la función corporal. ${ }^{(17,26)}$

\section{Tamizaje propuesto}

Pese a la heterogeneidad de la literatura, dada la relación de la composición corporal con la funcionalidad y la función metabólica, en el contexto de obesidad 
sarcopénica se han propuesto distintos abordajes.
${ }_{27,28,7}, 7-9,10$,

En cuanto al índice empleado en la determinación de obesidad sarcopénica, dos ensayos clínicos sugieren que el uso de la masa muscular apendicular en relación con el peso permite una mejor detección de sarcopenia en pacientes con sobrepeso u obesidad y a su vez permiten una mejor aproximación diagnóstica en relación al riesgo metabólico. $(10,28)$

Se puede aplicar un algoritmo para el tamizaje de esta entidad, que puede incluir los siguientes criterios acuñados en la literatura. Ver figura 1 y $2^{(2,4,7-9,28)}$

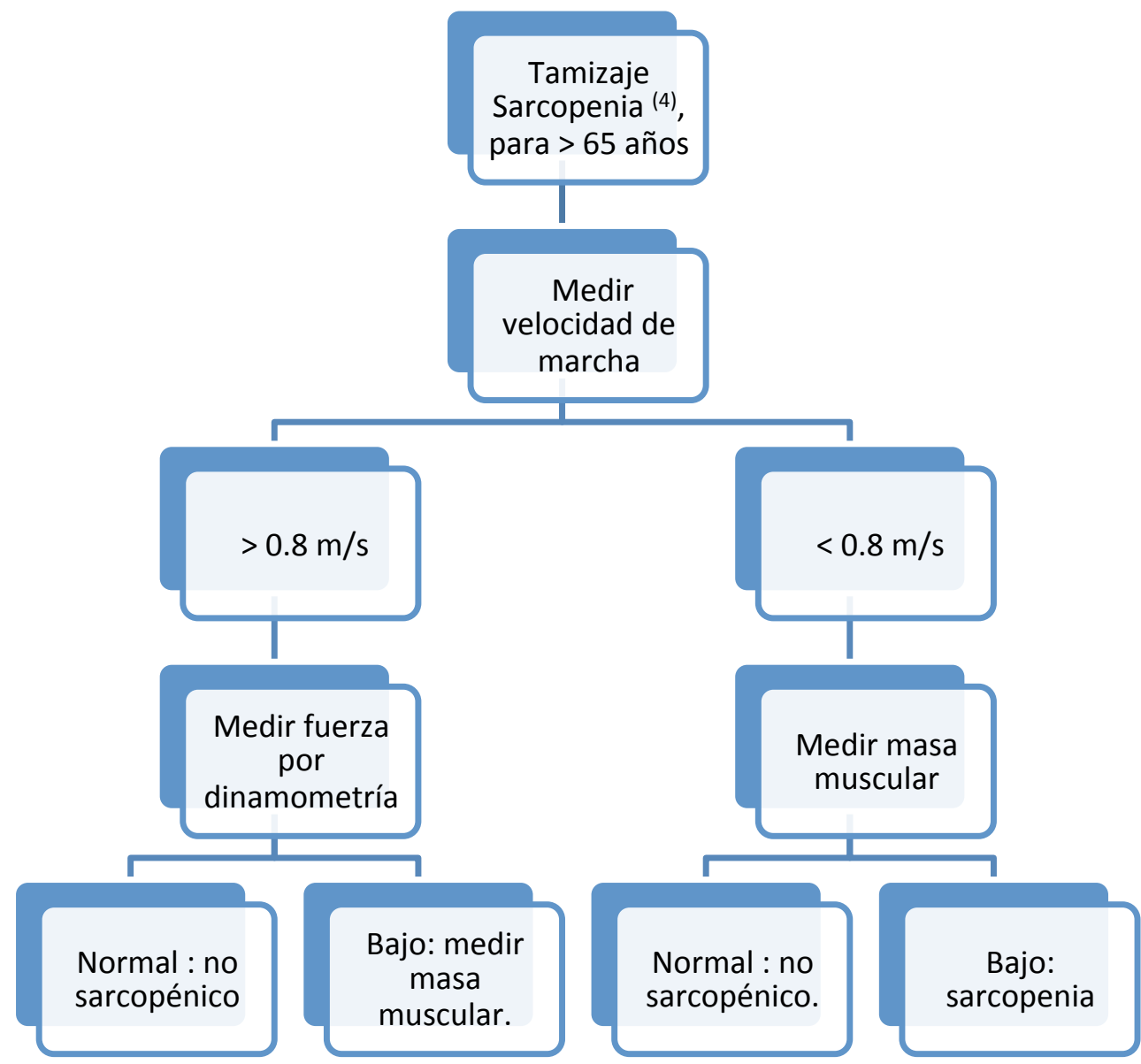

Figura 1 Propuesta de abordaje diagnóstico de sarcopenia, modificado de referencias 4.

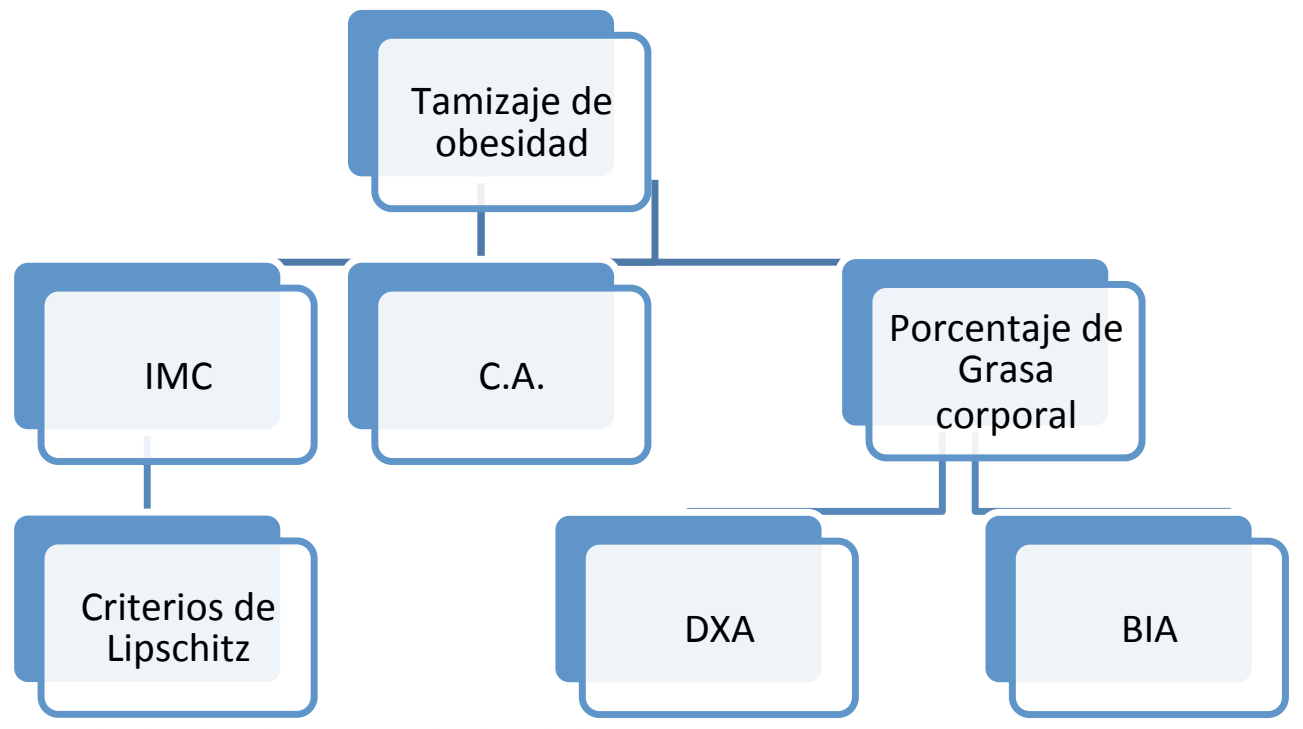

Figura 2 Propuesta de abordaje diagnóstico de obesidad, según referencias 8 y 28 


\section{CONCLUSIONES}

La obesidad sarcopénica es una entidad con importantes implicaciones sistémicas, donde el cambio de composición corporal, afecta tanto la funcionalidad del músculo como el riesgo cardiovascular y metabólico.

El abordaje de la fisiología del tejido muscular y adiposo ha permitido entender sus interacciones y el desbalance entre factores anabólicos y catabólicos así como de la inflamación como importante mediador entre ambos procesos. El impacto, sólo a nivel de biomecánico, se ha determinado que puede darse una tasa anual de pérdida de fuerza isométrica de hasta 1 a $2 \%$, mientras que en el componente de poder, definido como la relación de velocidad de acortamiento y fuerza, de hasta un 3 a $4 \%$. (19)

Con el estudio de Helsinsky que define el riesgo en función de los cambios de composición corporal en forma longitudinal, se sugiere que las intervenciones para mantener una composición corporal basada en el riesgo metabólico están justificadas, el adulto mayor que no tenga condición de fragilidad. Por tanto, el diagnóstico de obesidad sarcopénica permite detectar indicadores a nivel subclínico o clínico de alto riesgo y su manejo tiene gran potencial respecto a la atenuación de la morbilidad y mortalidad en el adulto mayor.

\section{AGRADECIMIENTO ESPECIAL}

A la Dra Isabel Barrientos, médico especialista en Geriatría y Gerontología, en el Hospital Nacional de Geriatría y Gerontología Dr. Raúl Blanco Cervantes, miembro del comité de soporte nutricional. La Dra Barrientos desde su labor docente motivó la presente revisión y ésta fue posible gracias a su aporte como experta en la materia.

\section{DECLARACIÓN DE CONFLICTO DE INTERESES}

Ninguno de los autores de la presente revisión tiene conflictos de intereses.

\section{BIBLIOGRAFÍA}

1. Fielding RA, Vellas B, Evans WJ, y col. Sarcopenia: An Undiagnosed Condition in Older Adults. Current Consensus Definition: Prevalence, Etiology, and Consequence; J Am Med Dir Assoc.; 2011; 12(4): 249-256. (Manuscrito del autor consultado en PubMed Central $\left.{ }^{\circledR}\right)$

2. KimY; Chung Y; Lee D; $y$ col. Prevalence of Sarcopenia and Sarcopenic Obesity in the Korean Population Based on the Fourth Korean
National Health and Nutritional Examination Surveys. J Gerontol A BiolSci Med Sci.; 2012;67(10):1107-1113

3. Baumgartner R; Koehler K; Gallagher D; y col. Epidemiology of Sarcopenia among the Elderly in New Mexico Am.J. Epidemiol; 1998;147:755-63.

4. Cruz-Jentoft AJ, Baeyens JP, Bauer JM, y col. Sarcopenia: European consensus on definition and diagnosis: Report of the European Working Group on Sarcopenia in Older People. Age Ageing; 2010;39(4):412-23.

5. Abellan Van Kan G., Cedarbaum J.M.,Cesari M; y col. Sarcopenia: Biomarkers And Imaging (International Conference On Sarcopenia Research) The Journal of Nutrition, Health \& Aging. 2011; 10: 834:846

6. Donin L.M; Poggiogalle E; Migliaccio S; $y$ col. Body composition in sarcopenic obesity: systematic review of the literature Mediterr. J. Nutr.Metab. 2013;6:191-198.

7. Mathus-Vliegen E.M. Obesity and the Elderly J.Clin. Gastroenterol; 2012;46:533-544.

8. Schaap L; Koster A; Visser M (2013) Adiposity, Muscle Mass, and Muscle Strength in Relation to Functional Decline in Older Persons Epidemiologic Reviews 2013

9. Zoico E, Francesco V, Guralnik JM, y col (2004) Physical disability and muscular strength in relation to obesity and different body composition indexes in a sample of healthy elderly women International Journal of Obesity 28, 234-241

10. Domiciano D;.Figueiredo C, Lopes J; OsteoporosInt (2013) 24:595-603 DOI 10.1007/s00198-012-2002-1 Discriminating sarcopenia in community-dwelling older women with high frequency of overweight/obesity: the São Paulo Ageing \& Health Study (SPAH)

11. Lipschitz DA. (1994) Screening for nutritional status in the elderly. Prim Care. ;21:55-67

12. Kelly TL, Wilson KE, Heymsfield SB (2009) Dual Energy X-Ray Absorptiometry Body Composition Reference Values from NHANES. PLoS ONE 4(9): e7038. doi:10.1371/journal.pone.0007038

13. Baumgartner RN, Wayne SJ, Waters DL, y col. (2004) Sarcopenic obesity predicts instrumental activities of daily living disability in the elderly. Obes Res.;12:1995-2004.

14. EPIDOS Rolland Y, Lauwers-Cances V, Cristini C, y col (2009) Difficulties with physical function associated with obesity, sarcopenia, and sarcopenic-obesity in community-dwelling elderly women: the EPIDOS (EPIDemiologie de l'OSteoporose) Study. Am J Clin Nutr;89:1895-900

15. Gómez-Cabello A; G. Rodríguez V; VilaMaldonado S; y col;(2012) Revisión, 
Envejecimiento y composición corporal: la obesidad sarcopénica en España Nutr Hosp.;27(1):22-30

16. Kohara K, (2013)Sarcopenic obesity in aging population: current status and future directions for research Endocrine. DOI 10.1007/s12020013-9992-0

17. Janssen I, Heymsfield SB, Ross R (2002). Low relative skeletal muscle mass (sarcopenia) in older persons is associated with functional impairment and physical disability. J Am Geriatr Soc; $50: 889$ - 896

18. Prado C, Wells J, Smith SR;et al (2012) Review Sarcopenic obesity: A Critical appraisal of the current evidence Clinical Nutrition 31 $583 \mathrm{e} 601$

19. Narici M; Maffulli N; (2010) Sarcopenia: characteristics, mechanisms and functional significance. British Medical Bulletin 2010;95: 139-15

20. StenholmS ., Harris T, Rantanen., Sarcopenic obesity - definition, etiology and consequences CurrOpinClinNutrMetab Care. 2008 November ; 11(6): 693-700

21. Goodpaster B, Park S, HarrisT, y col (2006)The Loss of Skeletal Muscle Strength, Mass, and Quality in Older Adults: The Health,Aging and Body Composition Study. Journal of Gerontology: MEDICAL SCIENCES 2006, Vol. 61A, No. 10, 1059-1064

22. Kohara K, Ochi M, Tabara Y, Nagai T, Igase M, $y$ col. (2011) Leptin in Sarcopenic Visceral Obesity: Possible Link between Adipocytes and Myocytes. PLoS ONE 6(9): e24633. doi:10.1371/journal.pone.0024633

23. Migliaccio, S Francomano D; Bruzziches R; $y$ col, (2013)Trunk Fat Negatively Influences Skeletal and Testicular Functions in Obese Men: Clinical Implications for the Aging Male International Journal of Endocrinologyconsultado en 15 de enero de 2013en http://dx.doi.org/10.1155/2013/18275

24. Reynolds S; Recent Developments in Obesity Research: Linkages between Obesity, Disability, and Physical Functioning CurrObes Rep.2013;2:267-274.

25. Abbatecola A.M; Chiodini P; Gallo C; y col Pulse wave velocity is associated with muscle mass decline: Health ABC study AGE. 2012;34:469-478

26. Strandberg T; Stenholm S; Strandberg A; y col (2013) The"ObesityParadox,"Frailty, Disability, and Mortality in Older Men: A Prospective, Longitudinal Cohort Study Am J Epidemiol. 2013;178(9):1452-1460

27. Lim S; KimJ; YoonJ; y col Sarcopenic Obesity: Prevalence and Association With Metabolic Syndrome in the Korean Longitudinal
Study on Health and Aging (KLoSHA) Diabetes Care; 2010;33:1652-1654.

28. Waters D; Baumgartner R, Sarcopenia and Obesity ClinGeriatr Med 2011;27: 401-421 